\title{
THE
}

\section{Self-Injurious Thoughts and Behaviors Among College Students: A Latent Class Analysis}

\author{
Marisa E. Marraccini
}

A. Brick

Conor O'Neill

Lisa L. Weyandt

University of Rhode Island, lisaweyandt@uri.edu

Ashley L. Buchanan

University of Rhode Island, buchanan@uri.edu

Follow this and additional works at: https://digitalcommons.uri.edu/php_facpubs

The University of Rhode Island Faculty have made this article openly available.

Please let us know how Open Access to this research benefits you.

This is a pre-publication author manuscript of the final, published article.

Terms of Use

This article is made available under the terms and conditions applicable towards Open Access Policy Articles, as set forth in our Terms of Use.

\section{Citation/Publisher Attribution}

Marisa E. Marraccini, Leslie A. Brick, J. Conor O’Neill, Lisa L. Weyandt \& Ashley L. Buchanan (2020) SelfInjurious Thoughts and Behaviors Among College Students: A Latent Class Analysis, Archives of Suicide Research, DOI: $10.1080 / 13811118.2020 .1746942$

Available at: https://doi.org/10.1080/13811118.2020.1746942 


\section{LATENT CLASS ANALYSIS OF COLLEGE STUDENT SITB}

Self-Injurious Thoughts and Behaviors Among College Students: A Latent Class Analysis Submitted to: Archives of Suicide Research

Marisa E. Marraccini, PhD, ${ }^{\mathrm{a}}$ Leslie A. Brick, PhD, ${ }^{\mathrm{b}} \mathrm{J}$. Conor O’Neill, $\mathrm{PhD},{ }^{\mathrm{c}}$ Lisa L. Weyandt, PhD, ${ }^{\text {d Ashley L. Buchanan, DrPH, MS }}{ }^{\mathrm{e}}$

${ }^{\text {a }}$ School of Education, University of North Carolina at Chapel Hill; ORCID ID: 0000-00027965-3703

${ }^{b}$ Department of Psychiatry and Human Behavior, Alpert Medical School of Brown University

${ }^{\mathrm{c}}$ Department of Psychiatry and Behavioral Sciences, Duke University School of Medicine

${ }^{\mathrm{d}}$ Department of Psychology, Interdisciplinary Neuroscience Program, University of Rhode Island

${ }^{\text {e }}$ Department of Pharmacy Practice, College of Pharmacy, University of Rhode Island

\section{Author Note}

The project described in this manuscript was supported by the National Center for Advancing Translational Sciences (NCATS), National Institutes of Health, through Grant Award Number UL1TR002489. The content is solely the responsibility of the authors and does not necessarily represent the official views of the NIH.

Correspondence concerning this article should be addressed to Marisa Marraccini, School of Education, CB 3500, University of North Carolina at Chapel Hill, Chapel Hill, NC. E-mail: mmarracc@email.unc.edu.

No potential conflict of interest was reported by the authors. 


\title{
LATENT CLASS ANALYSIS OF COLLEGE STUDENT SITB
}

\begin{abstract}
This study explored emerging typologies of self-injurious thoughts and behaviors and how they relate to known psychological and cognitive correlates. Latent class analysis was employed to identify subgroups of college students $(n=626$, mean age $=20,73 \%$ female, and $83 \%$ White) based on history of self-injurious thoughts and behaviors. Differences among classes were tested to examine their associations with suicidal ideation, fearlessness of death, depression/hopelessness, thwarted belongingness, problems with alcohol or drugs, behavioral inhibition, and emotional control. Results of the latent class analysis supported a 3-class solution, with students classified as being likely to have (1) no history of any self-injurious thoughts and behaviors (78\%); (2) a history of all self-injurious thoughts and behaviors (8\%); or (3) a history of suicidal ideation, plan, and non-suicidal self-injurious thoughts and behaviors, but not suicide attempt (13\%). Findings examining correlates of self-injurious thoughts and behaviors highlight the importance of fearlessness of death for distinguishing suicide attempters from nonattempters. Attention to mental health and suicide prevention initiatives should remain a critical priority across college campuses.
\end{abstract}

Keywords: self-injurious thoughts and behaviors; suicide; college students; latent class analysis. 


\section{LATENT CLASS ANALYSIS OF COLLEGE STUDENT SITB}

Self-injurious thoughts and behaviors (SITB) remain a critical public health issue among young adults and college aged individuals. SITB include both suicidal (ideation, plans, attempts) and non-suicidal self-injurious (NSSI) thoughts and behaviors (Nock, Prinstein, \& Sterba, 2009), with NSSI defined as self-injury without the intent to die (Nock, Holmberg, Photos, \& Michel, 2007). It is estimated that one out of five (22.3\%) college students experiences suicidal ideation, and 3.2\% have made a suicide attempt (Mortier et al., 2018). Among college-aged individuals, prevalence rates of NSSI are estimated to be $13.4 \%$ (Swannell, Martin, Page, Hasking, \& St. John, 2014), with NSSI and suicide attempts frequently co-occurring (Hamza, Stewart, \& Willoughby, 2012). All types of SITB, including NSSI (Klonsky, May, \& Glenn, 2013; Ribeiro et al., 2016), confer elevated risk for suicide for both men and women; however, prevalence estimates are generally higher among women (Mortier et al., 2018).

Correlates of SITB include a range of psychological symptoms, cognitive markers, and environmental factors. Some of these may include depression, hopelessness, drug or alcohol use disorders, anxiety, attention-deficit hyperactivity disorder (ADHD), and emotion dysregulation (Balazs \& Kereszteny, 2017; Esposito, Spirito, Boergers, \& Donaldson, 2003; Klonsky, Qui, \& Saffer, 2016; May \& Klonsky, 2016; Zlotnick, Donaldson, Spirito, \& Pearlstein, 1997). Although differences in depression, anxiety, and hopelessness between ideators (individuals with suicidal ideation) and attempters (individuals who have previously made a suicide attempt) are relatively small (May \& Klonsky, 2016), a growing body of research suggests significantly worse outcomes related to drug and alcohol use disorders and emotion dysregulation among suicide attempters (Esposito et al., 2003; May \& Klonsky, 2016). Some of the key correlates of NSSI specifically include emotion dysregulation (Gratz \& Tull, 2010; Gratz \& Roemer, 2008), impulsivity (Hamza, Willoughby, \& Heffer, 2015; Liu et al., 2017; Victor \& Klonsky, 2014), 


\section{LATENT CLASS ANALYSIS OF COLLEGE STUDENT SITB}

and depression and anxiety (Taliaferro \& Muehlenkamp, 2015a; 2015b). Furthermore, multiple studies indicate that many of the psychological correlates of SITB appear to be worse for suicide attempters compared to those with NSSI (Hamza \& Willoughby, 2013).

Although most individuals with suicidal thoughts or NSSI behaviors do not attempt suicide, the majority of research fails to elucidate why some individuals move from ideation or NSSI to making a suicide attempt (Hamza et al., 2012; Klonsky et al., 2015). Multiple theories have attempted to explain this ideation-to-action framework, including the integrated motivational-volitional (IMV) theory, Joiner's Interpersonal Theory of Suicide (IPT), and the three-step theory of suicide (Dhingra et al., 2016; Klonsky \& May, 2015; Van Orden et al., 2010). The IMV theory suggests that individuals move from ideation-to-action across phases: a pre-motivational phase inclusive of background factors and triggering events, a motivational phase describing the formulation of suicidal ideation and intent, and a volitional phase that explains how individuals shift from ideation to attempt. The latter two phases are theorized to be moderated by distinct factors contributing to the development of suicidal desire and transition to suicidal behavior, respectively (O’Connor \& Kirtley, 2018).

Per IPT, suicidal ideation is posited to stem from a feeling of hopelessness about an experienced state of thwarted belongingness (i.e., loneliness, social isolation) and perceived burdensomeness to others (Van Orden et al., 2010). In order for an individual to attempt suicide, however, an individual must also acquire the capability to do so through experiences such as self-injury that increase pain tolerance and reduce the innate fear of death (Van Orden et al., 2010). Similarly, the three-step theory of suicide provides a broad framework that postulates that ideation results from the combination of pain and hopelessness (Klonsky \& May, 2015). Connectedness, or a sense of interpersonal closeness to others (Whitlock, Wyman, \& Moore, 


\section{LATENT CLASS ANALYSIS OF COLLEGE STUDENT SITB}

2014), is hypothesized to moderate the intensity of ideation, and the capability to attempt suicide is derived from dispositional (e.g., low pain tolerance), acquired (e.g., experiences shaping fearlessness of death), and practical factors (e.g., access to lethal means). Together, these capability factors contribute to the transition from ideation to attempt and one's capacity to engage in suicidal behavior (Klonsky \& May, 2015).

Empirical investigations that distinguish suicidal patterns of behavior and elucidate risks separately for each outcome have critically important clinical implications (Klonsky et al., 2016; May \& Klonsky, 2016). For example, understanding differing risks for individuals considering suicide versus those preparing to act on suicide informs clinical decisions about treatment and crisis prevention (May \& Klonsky, 2016). Person-centered methodological approaches have emerged as a useful tool for disentangling patterns of SITB and associated risk factors (Martin, LaCroix, Novak, \& Ghahramanlou-Hollowy, 2019). Model based approaches such as Latent Class Analysis (LCA), which leverages heterogeneity among individuals, have been used to distinguish patterns of NSSI based on frequency and severity (Klonsky \& Olino, 2008; Peterson, Chen, Karver, \& Labouliere, 2019) and to better understand individuals engaging in NSSI most at risk for suicidal behavior (Hamza and Willoughby, 2013). Research conducted by Hamza and Willoughby (2013) suggests that compared to individuals classified by NSSI without suicidal thoughts and behaviors, individuals classified by NSSI and suicidal thoughts and behaviors experience more severe emotion dysregulation, depression, and behavioral disinhibition.

Although Hamza and Willoughby's (2013) study sheds light on how nonsuicidal and suicidal behaviors may overlap, only a single measure of suicidal behavior was included and other indicators of SITB such as plans or thoughts of NSSI were not addressed. More recently, Dhingra, Boduszek, and Klonsky (2016) classified individuals based on patterns of SITB that 


\section{LATENT CLASS ANALYSIS OF COLLEGE STUDENT SITB}

included ideation, plans, gestures, attempts, thoughts of NSSI, and NSSI. Results from the LCA, conducted on data from a sample of 1,809 university students in the United Kingdom, characterized students as falling in a low SITB group (45.8\%), a high suicidal ideation and NSSI group (25\%), or a high SITB group (29.9\%). Although individuals in the high SITB group and the high suicidal ideation and NSSI group reported significantly lower levels of belongingness than the low SITB group, only those in the high SITB group reported significantly higher levels of perceived burdensomeness and fearlessness of death compared to the other groups. These preliminary findings support that correlates of suicide need to be differentiated based on SITB type (May \& Klonsky, 2016), with factors related to acquired capability for suicide potentially accounting for differences in typologies (Klonsky et al., 2016; Hamza et al., 2012).

\section{The Current Study}

The current study was informed by researcher and clinician recommendations for studies to better differentiate typologies of SITB (Dhingra et al., 2016; Nock et al., 2008) and separately examine associated risk factors of SITB (e.g., Klonsky et al., 2013; May \& Klonsky, 2016). Dhingra and colleagues (2016) attempted to address this issue by conducting LCA with multiple types of SITB; however, their study was conducted in the UK and included a sample with an unusually elevated rate of suicidal ideation (over 60\%). Therefore, the current study attempts to differentiate typologies of SITB and further examine how emerging typologies of SITB relate to previously identified psychological and cognitive correlates of SITB in a sample of United States (US) college students.

The present study addressed two specific aims. First, this study aimed to estimate subgroups based on history of SITB (suicidal ideation, plans, attempts, thoughts of NSSI, and NSSI). Based on previous research (Dhingra et al., 2016), we hypothesized that classifications 


\section{LATENT CLASS ANALYSIS OF COLLEGE STUDENT SITB}

would be primarily be distinguished based on ideation and plans, attempts, and NSSI (both thoughts and behaviors). Second, this study aimed to explore the external validity of these classifications by examining how emerging SITB typologies relate to current suicide risk and previously established psychological and cognitive correlates of SITB. We hypothesized that, compared to individuals with low probabilities for reporting a history of any type of SITB, those with high probabilities for any type of SITB are more likely to report higher severity of recently endorsed suicidal ideation and impulses, as well as poorer functioning across other measures of psychological and cognitive functioning.

Considering that previous research elucidates important differences in psychological and cognitive functioning based on type of SITB, we specifically hypothesized that individuals classified in groups with risk for suicide attempts would report greater severity in psychological correlates and poorer functioning in cognitive correlates of SITB than other groups of maladaptive typologies. Consistent with IPT, we also hypothesized that individuals classified based on their history of suicide attempts based on LCA would report higher levels of fearlessness of death, and those classified based on previous attempts or NSSI would endorse greater impairments related to impulsivity and emotion dysregulation.

\section{Materials and Methods}

\section{Participants and Procedures}

Undergraduate students from two public universities in the United States, one in the southeast and one in the northeast, were recruited to participate in an online survey as part of a larger study concerning prescription opioid use. The email addresses of a random sample of 4,000 students were provided by each university's Registrar office, with a total of 8,000 students across both institutions invited to participate. Emails inviting students to participate in a larger 


\section{LATENT CLASS ANALYSIS OF COLLEGE STUDENT SITB}

study related to prescription opioid use among college students were sent over two phases during the spring 2018 semester. Informed consent and self-report surveys were available through REDCap, an electronic data collection system (Harris et al., 2009). Participants completed a battery of self-report measures as part of the larger study that was approved by each university's Institutional Review Board.

A total of 888 participants consented into the study (11\% response rate). After removing 16 graduate students and 246 participants with incomplete data for the study's primary measures related to suicidal thoughts and behaviors, the final analytical sample included $\mathrm{N}=626$ undergraduate students. The resulting participants were between the ages of 18 and $24(19.1 \%$ were $18,24.0 \%$ were $19,22.7 \%$ were $20,24.6 \%$ were $21,14.9 \%$ were $22,2.6 \%$ were 23 , and $1.3 \%$ were 24$)$. On average, they were 20 years of age and predominantly female $(72.7 \%)$ and white $(82.6 \%)$.

\section{Measures}

Demographic variables. Questions about participant demographic characteristics included university, self-reported sex, race, ethnicity, and age. Sex (male or female) was based on sex assigned at birth according to the information on participants' original birth certificates. The sample was predominantly white; therefore, non-Latinx, race and ethnicity were dichotomized (white or student of color, Latinx or non-Latinx, respectively).

Self-injurious thoughts and behaviors. The Self-Injurious Thoughts and Behaviors Interview (SITB; Nock et al., 2007) was administered to measure presence, frequency, and severity of SITB. The measure has strong psychometric support (Nock et al., 2007) and has been used as a self-report questionnaire in previous research (Dhingra et al., 2016). The dichotomous 


\section{LATENT CLASS ANALYSIS OF COLLEGE STUDENT SITB}

(yes/no) questions addressing lifetime history of suicidal ideation, plans, attempts, thoughts of NSSI, and NSSI were analyzed in the current study. Furthermore, considering the low prevalence of all attempts (suicide attempts [1.8\%], aborted attempts [5.0\%], and interrupted attempts [3.1\%]), we collapsed responses across all three attempt categories to include one indicator of lifetime history of any attempt including aborted or interrupted attempts.

Suicide Risk Correlates. Frequency and intensity of suicidal ideation and impulses over the past two weeks was measured with the Depressive Symptom Index: Suicidality Subscale (DSI-SS; Joiner, Pfaff, \& Acres, 2002). The DSI-SS is a brief, four-item self-report questionnaire with recommended clinical cut-off scores to assess risk and adequate reliability and validity, with convergence between suicidality and depressive symptoms and presenting complaint (Joiner et al., 2002). Respondents were instructed to select from four statements falling on a Likert-style scale that best describes four different types of ideation (during the past two weeks): thoughts of killing oneself, thoughts about suicide as it relates to a suicide plan, thoughts of suicide as it relates to control, and impulses to kill oneself. For example, statements related to thoughts of killing oneself range from 0 ("I do not have thoughts of killing myself") to 3 ("I always have thoughts of killing myself"). In the present study, internal reliability using Cronbach's alpha was adequate $(\alpha=.89)$ across the four items.

Fearlessness of death was measured with the Acquired Capability for Suicide ScaleFearlessness About Death (ACSS-FAD), which has demonstrated good convergent and divergent validity (Ribeiro et al., 2014). This measure includes seven items about fearlessness of death with response options on a Likert scale from 0 ("not at all like me") to 4 ("very much like me"). Example items include "The fact that I am going to die does not affect me" and "The prospect of 


\section{LATENT CLASS ANALYSIS OF COLLEGE STUDENT SITB}

my own death arouses anxiety in me." In the present study, internal reliability was adequate $(\alpha=.76)$ across the seven items.

Psychological Correlates. To measure cross-cutting symptoms of mental health symptoms (i.e., depression, anxiety, substance use), we included the DSM-V Cross-Cutting Symptoms Measure (American Psychiatric Association, 2013). This dimensional measure was developed by the Diagnostic and Statistical Manual of Mental Disorders (DSM)-V Task Force and Work Groups to assess the presence and severity of symptoms cutting across multiple psychiatric diagnoses (Clarke \& Kuhl, 2014). Participants rated the frequency of being bothered by problems over the past two weeks, with response options rated on a Likert scale from 0 ("None, not at all") to 4 ("Severe, nearly every day"). The following items from this measure were included in the current analyses: depressed mood or hopelessness ("feeling down, depressed, or hopeless"), thwarted belongingness ("not feeling close to other people or enjoying your relationships with them"), alcohol (“drinking at least 4 or more drinks of any kind of alcohol in a single day"), and drug use ("using medicines on your own, that is, without a doctor's prescription, in greater amounts than prescribed, or drugs like marijuana, cocaine or crack, etc.").

Cognitive Correlates. The Behavior Rating Inventory of Executive Function-Adult Version (BRIEF-A), which measures adult executive function and self-regulation (Roth \& Gioia, 2005), was administered to measure emotional control and behavioral inhibition. Response options fall on a 3-point Likert scale from 1 ("Never") to 3 (“Always"). The emotional control subscale includes items such as "I have emotional outbursts for little reason" and "my mood changes frequently." The behavioral inhibition subscale includes items such as "I am impulsive" or "I make decisions that get me into trouble (legally, financially, socially)." Internal reliability 


\section{LATENT CLASS ANALYSIS OF COLLEGE STUDENT SITB}

in the present study was adequate ( $\alpha=.91$ for emotional control and $\alpha=.77$ for behavioral inhibition).

\section{Data Analysis}

Data management and descriptive analyses were conducted in SPSS Version 26 (IBM Corp., 2019) and Latent Class Analysis (LCA) was conducted in MPlus Version 8 using (Muthén \& Muthén, 1998-2017). The proportion of missing values pertaining to the measures included in the analytic sample $(n=626)$ ranged from $0 \%$ (age and sex) to $20.8 \%$ (fearlessness of death). Analyses were conducted using the full information maximum likelihood approach with robust standard errors in Mplus.

This study aimed to model how different patterns of SITB may work in conjunction to confer risk for suicide, and also understand how correlates of SITB may confer risk for these patterns. We therefore employed LCA using dichotomous lifetime SITB variables of (1) suicidal ideation, (2) suicide plan, (3) any suicide attempt (including aborted and interrupted), (4) thoughts of NSSI, and (5) NSSI. LCA is a model-based, data reduction method for identifying latent subgroups within a population based on response patterns among a set of variables and is comprised of two main sets of parameters: 1) Class membership probabilities (i.e., the proportion of individuals estimated to fall within each latent class); and 2) Item-response probabilities (i.e., representing the probability of endorsing a particular item, given class membership) (Collins \& Lanza, 2013).

Class enumeration was conducted to identify the optimal number of latent classes. Models comprised of 2-, 3-, 4-, and 5-classes were estimated and the following was considered: (1) Akaike's Information Criterion (AIC) and the sample-size adjusted Bayesian information criterion (SSA-BIC), with smaller values of the AIC and BIC indicating a better fit model 


\section{LATENT CLASS ANALYSIS OF COLLEGE STUDENT SITB}

(Akaike, 1987; Schwarz, 1978); (2) significance of Lo-Mendell-Rubin likelihood ratio test (LMR-LRT) and the Parametric Bootstrapped likelihood ratio test (BLRT), where a significant p-value indicates a better fitting model than the k-1 model, with $\mathrm{k}$ reflecting number of groups (McLachlan \& Peel, 2000); and (5) adequate entropy (with values approaching 1 indicating clear separation of classes) above .80 (Celeux \& Soromenho, 1996). We plotted class probabilities from each model to analyze theoretical and conceptual fit visually. To facilitate interpretations and comparisons, and considering the serious risk and low frequency of behaviors such as making a suicide attempt, item-response probabilities above .6 were considered "high" and probabilities below .1 were considered "low". Finally, the odds of correct classification $\left(\mathrm{OCC}_{\mathrm{k}}\right)$, which measures the certainty of the classifications of individuals, was calculated, with higher $\mathrm{OCC}_{\mathrm{k}}$ indicating a better model fit, and > 5 as desirable (Nagin, 2005). The average posterior class probability $\left(\mathrm{AvePP}_{\mathrm{k}}\right)$, which measures how well the model classifies individuals into the most likely class, was also measured to indicate latent class assignment error for each class $\operatorname{AvePP}_{\mathrm{k}}$ (>.70 indicates well-separated classes; Nagin, 2005). The final model was determined by a combination of theory, model fit, and diagnostic classification parameters.

To explore the validity of these typologies and understand how correlates of SITB may distinguish among classes, a number of auxiliary variables (referring to correlates outside of the measurement model) were included in the final model. Demographic variables (university, sex, race, ethnicity, and age) were examined as predictors of latent classes in a multinomial regression using the automatic three-step approach (i.e., AUXILLARY $=$ R3STEP). Next, the three-step approach was used to examine equality of means for suicide risk correlates (suicidal ideation/impulses, fearlessness of death), psychological correlates (depression and hopelessness, thwarted belongingness, problems with alcohol, and problems with drugs), and cognitive 


\section{LATENT CLASS ANALYSIS OF COLLEGE STUDENT SITB}

correlates (behavioral inhibition and emotional control) across latent classes. Based on recommendations by Asparouhov and Muthén (2014) for a three-step method of including auxiliary variables in mixture modeling, the setting DCATEGORICAL in MPlus was used to measure heterogeneity across categorical variables (Lanza et al., 2013) and BCH for continuous variables (Bakk \& Vermunt, 2016). Chi-square analyses and $p$-values were calculated to determine whether each class was significantly different from the other classes on each outcome variable.

\section{Results}

\section{Descriptive Analyses}

A total of 626 students completed the battery of self-report questionnaires related to SITB with data on all variables of interest. Lifetime reports of SITB variables and descriptive statistics for study variables are shown in Tables 1 and 2. Lifetime endorsement of SITB included: suicidal ideation (25.6\%), suicide plan(s) (7.7\%), suicide attempt(s) (including aborted or interrupted) (6.3\%), thoughts of NSSI (20.0\%), and NSSI (12.6\%). Bivariate correlations of key variables are shown in Table 3.

\section{Latent Class Analysis}

Model Fit and Classification Diagnostics. Table 4 displays information about the model fit and classification diagnostics used to select the most appropriate number of classes. The AIC, BIC, SSA-BIC, LMR-LRT, and BLRT all indicated preference for the 4-class solution; however, entropy was slightly higher for the 3- and 5-class solutions. Upon inspection of the class membership probabilities, the four-class solution contained a very small class size $(3.04 \%$, $\mathrm{n}=19$ ), which has potential to be spurious due to over-extraction of classes (Hipp \& Bauer, 2006). Further, the $\mathrm{AvePP}_{\mathrm{k}}$ for this class was 1.00, indicating perfect class assignment of 


\section{LATENT CLASS ANALYSIS OF COLLEGE STUDENT SITB}

individuals, possibly due to the small size of this group. While this class characterized a substantively meaningful group of participants that did not appear in the 3-class solution (e.g., individuals who were likely to endorse a lifetime history of suicidal ideation, suicide plans, suicide attempts, but were not likely to endorse items related to NSSI), we proceeded with interpretation of the 3-class model to avoid over-extraction (Hipp \& Bauer, 2006). Inspection of classification diagnostics for the 3-class model indicated good class-specific separation $\left(\right.$ AvePP $_{\mathrm{k}}$ $>.87$ and $\mathrm{OCC}_{\mathrm{k}}>14$; see Table 5). Visual inspection of the profile plot for the 3 -class solution is shown in Figure 1, where each indicator is plotted on the x-axis and the item-class probabilities are plotted on the y-axis.

Description of Latent Classes. Classes based on patterns of SITB are labeled according to probability for reporting a lifetime history of suicidal ideation, suicide plans, suicide attempts (including aborted and interrupted attempts), thoughts of NSSI and NSSI behaviors. The first class, labeled High SITB, included a group of students likely to report a history of suicidal ideation, suicide plans, and suicide attempts, as well as thoughts of NSSI and NSSI behavior. This class included $8.6 \%$ of the sample $(n=54)$. The second class, including $13.1 \%$ of the sample (n=82), was labeled High Suicidal Ideation/NSSI-Low Suicide Attempts (High SI/NSSI-Low SA) to reflect the group of students likely to report a history of suicidal ideation, as well as NSSI (thoughts and behaviors), but unlikely to report a history of suicide plans or attempts. The third class was labeled Low SITB as individuals in this group were unlikely to report any SITB. This group was the largest, with $78.3 \%$ of the sample $(n=490)$.

\section{Validation Analyses}

Demographic predictors of class membership. University (enrollment in northeast college or southeast college), sex (female or male), race (white or student of color), ethnicity 


\section{LATENT CLASS ANALYSIS OF COLLEGE STUDENT SITB}

(Latinx or non-Latinx), and age were examined as predictor variables of class membership.

Estimates from the multinomial regression are shown in Table 6. Only sex and age demonstrated significant differences across SITB classes. Females were significantly more likely to fall in either of the suicide risk classes, High SI/NSSI-Low SA or High SITB, compared to the Low SITB class. Additionally, older students were significantly less likely to fall in the High SITB class compared to the High SI/NSSI-Low SA class. Taken together, students who were younger or female were more likely to report a history of all SITB indicators compared to students who were older or male.

Model Validation with SITB Correlates. Table 7 displays the results of Chi-square tests examining differences in suicide risk, psychological correlates, and cognitive correlates across classes, described more thoroughly in the subsequent sections.

Suicide Risk. Level of suicidal ideation and impulses was significantly different between each of the three classes, with the highest levels reported in the High SITB class, the lowest levels reported in the Low SITB class, and those reported by the High SI/NSSI-Low SA classes falling in the middle. With regard to fearlessness of death, the class with the highest mean was High SITB, which was significantly higher than individuals falling in both the Low SITB class and the High SI/NSSI-Low SA class. The class with the lowest mean was the High SI/NSSI-Low $S A$, although statistically, it was not significantly lower than the Low SITB class.

Psychological Correlates. Individuals falling in the Low SITB class reported significantly lower levels of depression and hopelessness, as well as lower levels of thwarted belongingness, than those falling in any other class. No differences between levels of depression/hopelessness or thwarted belongingness emerged between the two high risk classes. 


\section{LATENT CLASS ANALYSIS OF COLLEGE STUDENT SITB}

With regard to substance use, no differences in alcohol use were observed, but individuals in the Low SITB class reported lower levels of illicit or nonmedical prescription drug problems compared with the High SITB class (but not High SI/NSSI-Low SA).

Cognitive Correlates. Individuals falling in the Low SITB class reported significantly lower severity of behavioral inhibition and emotional control than those falling in any other class. No significant differences between the two high risk classes emerged for behavioral inhibition and emotional control.

\section{Discussion}

The current study was among the first to systematically examine patterns of SITB among a general sample of college students from two universities located in the northeastern and southeastern regions of the US and explore the extent to which known markers for suicide risk and psychological and cognitive correlates associated with these patterns. Findings indicate that distinct typologies could be determined based on several indicators of history of suicidal and self-injurious thoughts and behaviors in a US based college population and that these typologies were related to differences in reports of current suicide risk, as well psychological and cognitive correlates.

\section{Patterns of SITB}

Three distinct and informative patterns of SITB were captured in our data. While the majority of students (78\%) fell in a group with a low probability for reporting any type of SITB, the remainder were classified as having a high probability for reporting all types of SITB (8\%), or a high probability for reporting suicidal ideation, thoughts of NSSI, and NSSI, but a low probability for reporting a suicide plan or attempt (13\%). These classifications are comparable to those reported by Dhingra and colleagues, whose LCA supported three groups of college 


\section{LATENT CLASS ANALYSIS OF COLLEGE STUDENT SITB}

students (a low SITB group, a high SITB group, and a high suicidal ideation and NSSI group).

Classifications reported by Dhingra et al., however, included a much lower number of students in the low risk group (46\%), and higher number among the two higher risk groups $(25 \%$ and $30 \%$, respectively), compared to the findings reported here. Differences may relate to the unusually high number of students reporting past SITB in Dhingra and colleagues' study compared to the current study's sample, which aligns more closely to prevalence estimates of SITB in the US college population (Mortier et al., 2018; Swannell et al., 2014).

\section{Validation of SITB Patterns and Correlates of SITB}

To validate the final classifications and explore differences in known correlates of these emerging typologies, the final model included (1) demographic variables, (2) measures of suicide risk (severity of suicidal ideation and impulsivity, fearlessness of death), (3) known psychological correlates of SITB, and (4) cognitive correlates of SITB. Overall, findings support the importance of severity of suicidal ideation and fearlessness of death in the ideation-to-action framework.

Demographic Differences. Among the demographic variables examined, only sex and age resulted in significant differences when comparing class membership probabilities. Female college students generally demonstrated higher probabilities for falling in the classes with high probabilities for reporting SITB (High SITB and High SI/NSSI-Low SA) than the low risk class. This finding is consistent with previous research that suggests that prevalence of suicidal ideation, plans, and attempts are generally higher among female college students than males (Balazs \& Kereszteny, 2017; Mortier et al., 2018).

Older students were also less likely to fall in the High SITB class compared to the High SI/NSSI-Low SA class. This finding is surprising considering self-report of SITB captured history 


\section{LATENT CLASS ANALYSIS OF COLLEGE STUDENT SITB}

of behaviors, expected to increase in prevalence with age, but may be due to a limited number of students representative of older ages (less than $4 \%$ of the sample were $23-24$ and more than $80 \%$ were 18-21). Although previous research suggests that retrospective measures of suicide attempts may be a valid method for measuring history of attempts in older adolescents (Mazza, Catalano, Abbott, \& Haggery, 2011), because this study did not follow students over time and this finding is based on the small number of students reporting SITB history, this finding should be interpreted cautiously. Future work investigating these relationships longitudinally is merited to better understand how these correlates may predict behaviors.

Suicide Risk. The finding that individuals falling in classes related to some risk for SITB (i.e., High SITB, High SI/NSSI-Low SA) demonstrated increased severity in suicide risk variables, as well as psychological and cognitive functioning, lends credibility to the validity of the typologies. Furthermore, because the High SITB and High SI/NSSI-Low SA classes are differentiated by the probability of a suicide attempt, findings related to differences in measures of suicide risk between these two high risk classes generally support a continuum of risk for understanding suicide. Thus, study results reinforce conventional theories of suicide including the IPT, IMV model, and three-step theory of suicide that identify fearless of death as a key pathway within the ideation-to-action framework (Dhingra et al., 2016; Klonsky \& May, 2015; Van Orden et al., 2010). Specifically, individuals in the High SITB class reported significantly higher severity on suicidal ideation and impulses than those in the High SI/NSSI-Low SA class, underscoring the significance of having a history of a suicide attempt(s) and its potential role for intensifying suicidal ideation. Indeed, research examining predictors of ideation and attempts has demonstrated distinct and shared risk factors with support for a continuum of risk (ten Have, van Dorsselar, \& de Graaf, 2013). Characteristics of suicidal ideation such as the age of onset, 


\section{LATENT CLASS ANALYSIS OF COLLEGE STUDENT SITB}

recurrence of ideation, and onset of plan have shown a positive association with suicide attempts in epidemiological samples, with prior suicide plans serving as the strongest predictor of attempts (ten Have et al., 2013). Reciprocally, individuals with a history of multiple suicide attempts appear to be at greater risk for recurrent suicidal ideation (Fuller-Thomson, West, \& Baiden, 2019; O’Connor \& Kirtley, 2018), which findings from the current study appear to support.

Because suicide attempts have been positively associated with fearlessness of death (Van Orden et al., 2008), with evidence that suicide attempters differ from ideators specifically with respect to their fear of death (Dhingra et al., 2015; Smith, Cukrowicz, Poindexter, Hobson, \& Cohen, 2010), we also hypothesized that individuals with patterns related to history of suicide attempt(s) to endorse higher levels of fearlessness of death. As expected, individuals falling in the High SITB class reported the highest levels of fearlessness of death, reinforcing fearlessness of death as a critical pathway within the ideation-to-action framework (Klonsky \& May, 2015; Van Orden et al., 2010).

Researchers have also posited that fearlessness of death emerges through habituation of the fear response by way of painful and provocative experiences, including NSSI (Klonsky et al., 2013; Van Orden et al., 2010). Interestingly, individuals with low probabilities for history of suicide attempt(s), but high probabilities for ideation and NSSI (High SI/NSSI-Low SA), reported the lowest levels of fearlessness of death. Because these reports were significantly lower than reports from individuals in the High SITB class, this finding intimates the possibility for fear of death to serve as a protective mechanism against suicidal behaviors among individuals with both suicidal ideation and NSSI behaviors, and may further support distinct pathways for suicide ideation and suicide attempts offered by conventional suicide theories (Dhingra, Boduszek, \& 


\section{LATENT CLASS ANALYSIS OF COLLEGE STUDENT SITB}

O’Connor, 2015; Klonsky \& May, 2015; Van Orden et al., 2010). The positive relationship between NSSI and suicide attempts overall (Klonsky et al., 2013) warrants further research examining fearlessness of death with individuals engaging in NSSI.

Psychological Correlates of SITB. Examination of differences in classes according to psychological correlates of SITB included problems with depression or hopelessness, thwarted

belongingness, alcohol, and drugs. As expected, reported problems with depression and thwarted belongingness were significantly higher among individuals falling in the high SITB classes (High SITB and High SI/NSSI-Low SA) compared to the Low SITB class. Differences for severity of depression and hopelessness between the High SITB and High SI/NSSI-Low SA classes, however, were not significant. This finding is inconsistent with prior research demonstrating that the severity of depression is associated with the severity of suicidal ideation as well as with suicide attempts (Van Orden, Lynam, Hollar, \& Joiner, 2006) and merits further investigation in the college population. The importance of current or recent SITB may be a necessary consideration, given the current study relied on lifetime histories that may or may not be relevant to present day experiences.

As expected, individuals classified in the High SITB class reported significantly higher levels of problems with drugs than those in the Low SITB class. Significant differences in frequency of problems with alcohol, however, did not emerge between any of the classes. This finding may relate to the normative alcohol use in a college population. Indeed, previous work examining the role of alcohol use as a risk factor for suicide in college students has largely supported no relationship between alcohol use and suicidal thoughts and behaviors, with researchers hypothesizing that the alcohol use is distinctly different among college populations 


\section{LATENT CLASS ANALYSIS OF COLLEGE STUDENT SITB}

compared to non-college adolescent and adult populations (e.g., Brownson, Drum, Smith, \& Burton Denmark, 2011; Gauthier, Witte, \& Correia, 2017).

Cognitive Correlates of SITB. As hypothesized, individuals falling in the Low SITB class reported significantly lower levels of problems related to emotional control and behavioral inhibition than those in all other classes. However, despite previous research that suggests individuals with a history of NSSI and a suicide attempt demonstrate significantly higher selfreport and performance measures of impulsivity compared to individuals with a history of NSSI but not suicide attempts (Dougherty et al., 2009), in our sample, neither emotional control nor behavioral inhibition significantly differentiated these behaviors. In other words, severity of emotional control and behavioral inhibition was comparable in the High SITB and High SI/NSSILow SA classes. Findings from a recent meta-analysis suggest that impulsivity may actually serve as a distal risk factor (as opposed to proximal) that increases the probability of engaging in experiences that reduce fearlessness of death and increase painful and provocative experiences, thereby increasing acquired capability (Anestis, Soberay, Gutierrez, Hernandez, \& Joiner, 2014). The present results offer preliminary support for this model given that the High SITB group demonstrated the highest levels of both fearlessness of death and impulsivity compared to the Low SITB group, though additional research examining the potential mediating effect of impulsivity across SITB typologies is indicated.

Limitations and Future Directions

The cross-sectional nature of this study, which relied on perceptions of lifetime history of SITB retrospectively, limits our understanding of current thoughts and behaviors and how these relate to current levels of functioning. Studies that use both current metrics and longitudinal designs will shed light on how these patterns may differentiate subsequent SITB as opposed to 


\section{LATENT CLASS ANALYSIS OF COLLEGE STUDENT SITB}

correlates of past SITB. Furthermore, the potential for reporting bias due to the self-report nature of this study and limitations concerning single item measures used for psychological correlates should be noted. Because this study does not adequately represent the population of all college students, findings may not generalize to students attending other universities. Furthermore, the low response rate of university students indicates the potential for selection bias among the participating sample.

Regarding the classifications, the decision to interpret the 3-class solution instead of the 4-class solution merits further investigation. Although we did not interpret the 4-class solution due to its small number of observations (Hipp \& Bauer, 2006), results indicated the possibility of a fourth class with substantive meaning: participants likely to report a history of suicidal thoughts and attempts, but unlikely to report NSSI. Because the current study relied on a convenience sample of unselected sample of college students of whom only $7.7 \%$ reported a lifetime suicide attempt, examination of patterns of SITB among a selected sample of college students with a history of SITB is warranted.

Despite these limitations, a relative strength of this study is the use of LCA, an approach that acknowledges individual heterogeneity. The elucidation of latent subgroups within a population can serve to inform our understanding of these individuals and help to identify unique characteristics that may lead to better prevention and treatment programs. Thus, findings from the current study underscore the importance of prioritizing mental health and suicide prevention on college campuses, with an emphasis on considering risk for suicide on a continuum. Implications and Conclusion

Clinicians supporting college students with a history of SITB should not only consider varying treatment approaches based on the different forms of self-injurious thoughts and 


\section{LATENT CLASS ANALYSIS OF COLLEGE STUDENT SITB}

behaviors, but also the potential for SITB to co-occur, treating risk for multiple types of SITB concomitantly. Because ongoing suicide risk appears to worsen from ideation-to-action (and in this case, potentially from history of self-injury and ideation to history of attempt), increasingly intensive supports and services should be considered across the continuum of suicidal and selfinjurious thoughts and behaviors. On a preventative level, screening initiatives and programs to promote campus engagement and social connectedness for students with a history of SITB could help to foster the well-being of these high-risk students. Results from this study underscore the pressing need to prioritize the mental health of college students and provide more intensive services for students arriving with previous SITB. 


\section{LATENT CLASS ANALYSIS OF COLLEGE STUDENT SITB}

\section{References}

Akaike, H. (1987). Factor analysis and AIC. In Selected Papers of Hirotugu Akaike (pp. 371386). Springer, New York, NY.

American Psychiatric Association. (2013). Diagnostic and statistical manual of mental disorders (DSM-5®). American Psychiatric Pub.

Anestis, M., Soberay, K., Gutierrez, P., Hernandez, T., \& Joiner, T. (2014). Reconsidering the link between impulsivity and suicidal behavior. Personality and Social Psychology, 18, 366-386.

Asparouhov, T., \& Muthén, B. (2014). Auxiliary variables in mixture modeling: Three-step approaches using M plus. Structural Equation Modeling: A Multidisciplinary Journal, 21, 329-341.

Bakk, Z., \& Vermunt, J. K. (2016). Robustness of stepwise latent class modeling with continuous distal outcomes. Structural Equation Modeling: A Multidisciplinary Journal, 23, 20-31.

Balazs, J., \& Kereszteny, A. (2017). Attention-deficit/hyperactivity disorder and suicide: A systematic review. World journal of psychiatry, 7, 44-59.

Brownson, C., Drum, D. J., Smith, S. E., \& Burton Denmark, A. (2011). Differences in suicidal experiences of male and female undergraduate and graduate students. Journal of College Student Psychotherapy, 25, 277-294.

Celeux, G., \& Soromenho, G. (1996). An entropy criterion for assessing the number of clusters in a mixture model. Journal of classification, 13(2), 195-212.

Clarke, D. E., \& Kuhl, E. A. (2014). DSM-5 cross-cutting symptom measures: a step towards the future of psychiatric care?. World Psychiatry, 13(3), 314. 


\section{LATENT CLASS ANALYSIS OF COLLEGE STUDENT SITB}

Collins, L.M., \& Lanza, S.T. Latent class and latent transition analysis with applications in the social, behavioral, and health sciences., volume 718. Hoboken, NJ: John Wiley \& Sons, 2013.

Dhingra, K., Boduszek, D., \& O’Connor, R. (2015). Differentiating suicide attempters from suicide ideators using the Integrated Motivational-Volitional model of suicidal behavior. Journal of Affective Disorders, 186, 211-218.

Dhingra, K., Boduszek, D., \& Klonsky, E. D. (2016). Empirically derived subgroups of selfinjurious thoughts and behavior: Application of latent class analysis. Suicide and LifeThreatening Behavior, 46, 486-499.

Dougherty, D., Mathia, C., Marsh-Richard, D., Prevette, K., Dawes, M., Hatzis, . . Nouvion, S. (2009). Impulsivity and clinical symptoms among adolescents with non-suicidal selfinjury with or without attempted suicide. Psychiatry Research, 169, 22-27.

Fuller-Thomson, E., West, K., \& Baiden, P. (2019) The ide does turn: Predictors of remission from suicidal ideation and attempt among Canadians who previously attempted suicide. Psychiatry Research, 274, 313-321.

Gauthier, J. M., Witte, T. K., \& Correia, C. J. (2017). Suicide ideation, alcohol consumption, motives, and related problems: exploring the association in college students. Suicide and Life-Threatening Behavior, 47, 142-154.

Gratz, K. L., \& Tull, M. T. (2010). The relationship between emotion dysregulation and deliberate self-harm among inpatients with substance use disorders. Cognitive therapy and research, 34, 544-553. 


\section{LATENT CLASS ANALYSIS OF COLLEGE STUDENT SITB}

Gratz, K. L., \& Roemer, L. (2008). The relationship between emotion dysregulation and deliberate self-harm among female undergraduate students at an urban commuter university. Cognitive behaviour therapy, 37, 14-25.

Esposito, C., Spirito, A., Boergers, J., \& Donaldson, D. (2003). Affective, behavioral, and cognitive functioning in adolescents with multiple suicide attempts. Suicide and LifeThreatening Behavior, 33, 389-399.

Hamza, C. A., Stewart, S. L., \& Willoughby, T. (2012). Examining the link between nonsuicidal self-injury and suicidal behavior: A review of the literature and an integrated model. Clinical psychology review, 32, 482-495.

Hamza, C. A., \& Willoughby, T. (2013). Nonsuicidal self-injury and suicidal behavior: A latent class analysis among young adults. PloS one, 8, e59955.

Hamza, C. A., Willoughby, T., \& Heffer, T. (2015). Impulsivity and nonsuicidal self-injury: A review and meta-analysis. Clinical psychology review, 38, 13-24.

Harris, P.A., Taylor, R., Thielke, R., Payne, J., Gonzalez, N., Conde, J.G. (2009). Research Electronic Data Capture (REDCap) - A metadata-driven methodology and workflow process for providing translational research informatics support. Journal of Biomedical Informatics, 42, 377-381.

Hipp, J. R., \& Bauer, D. J. (2006). Local solutions in the estimation of growth mixture models. Psychological Methods, 11, 36.

IBM Corp. Released 2019. IBM SPSS Statistics for Windows, Version 26.0. Armonk, NY: IBM Corp.

Joiner Jr, T. E., Pfaff, J. J., \& Acres, J. G. (2002). A brief screening tool for suicidal symptoms in adolescents and young adults in general health settings: reliability and validity data from 


\section{LATENT CLASS ANALYSIS OF COLLEGE STUDENT SITB}

the Australian National General Practice Youth Suicide Prevention Project. Behaviour research and therapy, 40, 471-481.

Klonsky, E. D., Qiu, T., \& Saffer, B. Y. (2016). Recent advances in differentiating suicide attempters from suicide ideators. Current opinion in psychiatry, 30(1), 15-20.

Klonsky, E.D., \& May, A. (2015). The three-step theory (3ST): A new theory of suicide rooted in the "ideation-to-action" framework. International Journal of Cognitive Therapy, 8, 114-129.

Klonsky, E.D., May, A., \& Glen, C. (2013). The relationship between nonsuicidal self-injury and attempted suicide: Converging evidence from four samples. Journal of Abnormal Psychology, 122, 231-237.

Klonsky, E. D., \& Olino, T. M. (2008). Identifying clinically distinct subgroups of self-injurers among young adults: a latent class analysis. Journal of consulting and clinical psychology, 76, 22.

Lanza, S. T., Tan, X., \& Bray, B. C. (2013). Latent class analysis with distal outcomes: A flexible model-based approach. Structural Equation Modeling, 20, 1-26.

Liu, R. T., Trout, Z. M., Hernandez, E. M., Cheek, S. M., \& Gerlus, N. (2017). A behavioral and cognitive neuroscience perspective on impulsivity, suicide, and non-suicidal self-injury: Meta-analysis and recommendations for future research. Neuroscience \& Biobehavioral Reviews, 83, 440-450.

Martin, J., LaCroix, J. M., Novak, L. A., \& Ghahramanlou-Holloway, M. (2019). Typologies of suicide: a critical literature review. Archives of suicide research, 1-16. 


\section{LATENT CLASS ANALYSIS OF COLLEGE STUDENT SITB}

May, A. M., \& Klonsky, E. D. (2016). What distinguishes suicide attempters from suicide ideators? A meta-analysis of potential factors. Clinical Psychology: Science and Practice, 23, 5-20.

Mazza, J. J., Catalano, R. F., Abbott, R. D., \& Haggerty, K. P. (2011). An examination of the validity of retrospective measures of suicide attempts in youth. Journal of Adolescent health, 49, 532-537.

McLachlan, G., \& Peel, D. (2000). Finite mixture models Hoboken, New Jersey: John Wiley \& Sons., Inc.

Mortier, P., Cuijpers, P., Kiekens, G., Auerbach, R. P., Demyttenaere, K., Green, J. G., ... \& Bruffaerts, R. (2018). The prevalence of suicidal thoughts and behaviours among college students: a meta-analysis. Psychological medicine, 48, 554-565.

Muthén, L.K., Muthén, B,O, 1998. Mplus User’s Guide, eight ed. Muthén \& Muthén, Los Angeles.

Myung, W., Han, C., Fava, M., Mischoulon, D., Papakostas, G., Heo, J-Y., . . Jeon, H. (2016). Reduced frontal-subcortical white matter connectivity in association with suicidal ideation in major depressive disorder. (2016). Translational Psychiatry, 6, 1-8.

Nagin D. Group-based modeling of development: Harvard University Press, 2005.

Nock, M. K., Borges, G., Bromet, E. J., Cha, C. B., Kessler, R. C., \& Lee, S. (2008). Suicide and suicidal behavior. Epidemiologic reviews, 30, 133-154.

Nock, M. K., Holmberg, E. B., Photos, V. I., \& Michel, B. D. (2007). Self-Injurious Thoughts and Behaviors Interview: development, reliability, and validity in an adolescent sample. Psychological Assessment, 19, 309-317. 


\section{LATENT CLASS ANALYSIS OF COLLEGE STUDENT SITB}

Nock, M. K., Prinstein, M. J., \& Sterba, S. K. (2009). Revealing the form and function of selfinjurious thoughts and behaviors: A real-time ecological assessment study among adolescents and young adults. Journal of abnormal psychology, 118, 816.

O'Connor, R. C., \& Kirtley, O. J. (2018). The integrated motivational-volitional model of suicidal behaviour. Philosophical Transactions of the Royal Society B: Biological Sciences, 373(1754), 20170268.

Peterson, A. L., Chen, J. I., Karver, M. S., \& Labouliere, C. D. (2019). Frustration with feeling: Latent classes of non-suicidal self-injury and emotion regulation difficulties. Psychiatry research, 275, 61-70.

Ribeiro, J.D., Franklin, J.C., Fox, K.R., Bentley, K.H., Kleiman, E.M., Chang, B.P., \& Nock, M.K. (2016) Self-injurious thoughts and behaviors as risk factors for future suicide ideation, attempts, and death: a meta-analysis of longitudinal studies. Psychological Medicine, 46, 225-236.

Ribeiro, J.D., Witte, T.K., Van Orden, K.A., Selby, E.A., Gordon, K.H., Bender, T.W., Joiner, T.E. (2014). Fearlessness about death: The psychometric properties and construct validity of the revision to the Acquired Capability for Suicide Scale. Psychol Assess. 26, 115-126.

Roth, R. M., \& Gioia, G. A. (2005). Behavior rating inventory of executive function--adult version. Lutz, FL: Psychological Assessment Resources.

Schwarz, G. (1978). Estimating the dimension of a model. The annals of statistics, 6(2), 461464.

Smith, P., Cukrowicz, K., Poindexter, E., Hobson, V., \& Cohen, L. (2010) The acquired capability for suicide: A comparison of suicide attempters, suicide ideators, and nonsuicidal controls. Depression and Anxiety, 27, 871-877. doi:10.1002/da.20701 


\section{LATENT CLASS ANALYSIS OF COLLEGE STUDENT SITB}

Swannell, S. V., Martin, G. E., Page, A., Hasking, P., \& St John, N. J. (2014). Prevalence of nonsuicidal self-injury in nonclinical samples: Systematic review, meta-analysis and meta-regression. Suicide and Life-Threatening Behavior, 44, 273-303.

Taliaferro, L. A., \& Muehlenkamp, J. J. (2015a). Factors associated with current versus lifetime self-injury among high school and college students. Suicide and Life-Threatening Behavior, 45, 84-97.

Taliaferro, L. A., \& Muehlenkamp, J. J. (2015b). Risk factors associated with self-injurious behavior among a national sample of undergraduate college students. Journal of American college health, 63, 40-48.

ten Have, M., van Dorsselar, S., \& de Graaf, R. (2013). Prevalence and risk factors for first onset of suicidal behaviors in the Netherlands Mental Health Survey and Incidence Study-2. Journal of Affective Disorders, 147, 205-211.

Van Orden, K. A., Lynam, M. E., Hollar, D., \& Joiner, T. E. (2006). Perceived burdensomeness as an indicator of suicidal symptoms. Cognitive Therapy and Research, 30, 457-467.

Van Orden, K. A., Witte, T. K., Cukrowicz, K. C., Braithwaite, S. R., Selby, E. A., \& Joiner Jr, T. E. (2010). The interpersonal theory of suicide. Psychological review, 117, 575.

Victor, S. E., \& Klonsky, E. D. (2014). Correlates of suicide attempts among self-injurers: A meta-analysis. Clinical Psychology Review, 34, 282-297.

Waldinger, R., Diguer, L., Guastella, F., Lefebvre, R., Allen, J., Luborsky, L., \& Hauser, S. (2002). The same old song? Stability and change in relationship schemas from adolescence to young adulthood. Journal of Youth and Adolescence, 31, 17-29. 


\section{LATENT CLASS ANALYSIS OF COLLEGE STUDENT SITB}

Whitlock, J., Wyman, P. A., \& Moore, S. R. (2014). Connectedness and suicide prevention in adolescents: Pathways and implications. Suicide and Life-Threatening Behavior, 44(3), 246-272.

Zlotnick, C., Donaldson, D., Spirito, A., \& Pearlstein, T. (1997). Affect regulation and suicide attempts in adolescent inpatients. Journal of the American Academy of Child \& Adolescent Psychiatry, 36, 793-798. 
LATENT CLASS ANALYSIS OF COLLEGE STUDENT SITB

Table 1

Frequencies of Demographic and SITB Variables

\begin{tabular}{|c|c|c|}
\hline & Frequency & $\%$ \\
\hline \multicolumn{3}{|l|}{ University } \\
\hline Southeast & 249 & 39.8 \\
\hline Northeast & 376 & 60.2 \\
\hline \multicolumn{3}{|l|}{ Sex } \\
\hline Female & 455 & 72.7 \\
\hline Male & 171 & 27.3 \\
\hline \multicolumn{3}{|l|}{ Lifetime SI } \\
\hline Yes & 160 & 25.6 \\
\hline No & 465 & 74.4 \\
\hline \multicolumn{3}{|l|}{ Lifetime SP } \\
\hline Yes & 48 & 7.7 \\
\hline No & 576 & 92.3 \\
\hline \multicolumn{3}{|l|}{ Lifetime SA } \\
\hline Yes & 38 & 6.3 \\
\hline No & 566 & 93.7 \\
\hline \multicolumn{3}{|c|}{ Lifetime NSSI thoughts } \\
\hline Yes & 118 & 20.0 \\
\hline No & 473 & 80.0 \\
\hline \multicolumn{3}{|l|}{ Lifetime NSSI } \\
\hline Yes & 79 & 12.6 \\
\hline No & 510 & 86.6 \\
\hline
\end{tabular}

Notes: NSSI = nonsuicidal self-injury; $\mathrm{SA}=$ suicide attempt, aborted attempt, or interrupted attempt; $\mathrm{SI}=$ suicidal ideation; $\mathrm{SITB}=$ selfinjurious thoughts and behaviors; $\mathrm{SP}=$ suicide plan. 
LATENT CLASS ANALYSIS OF COLLEGE STUDENT SITB

Table 2

Descriptive Statistics of Key Variables

\begin{tabular}{lcccc}
\hline & N & Mean (SD) & Response Range & Possible Range \\
\hline SI/impulse severity & 579 & $0.13(0.33)$ & $0-2$ & $0-3$ \\
Fearlessness of death & 496 & $2.06(0.86)$ & $0-4$ & $0-4$ \\
Depression/hopelessness & 410 & $1.0(1.06)$ & $0-4$ & $0-4$ \\
Thwarted Belongingness & 477 & $0.77(1.09)$ & $0-4$ & $0-4$ \\
Problems with alcohol & 477 & $0.61(0.85)$ & $0-4$ & $0-4$ \\
Problems with drugs & 479 & $0.31(0.84)$ & $0-4$ & $0-4$ \\
Behavioral inhibition & 622 & $1.34(0.33)$ & $1-2.9$ & $1-3$ \\
Emotional control & 624 & $1.31(0.39)$ & $1-2.9$ & $1-3$ \\
\hline
\end{tabular}

Emotional control

$1.31(0.39)$

$1-2.9$

standard deviation. 


\section{LATENT CLASS ANALYSIS OF COLLEGE STUDENT SITB}

\section{Table 3}

Bivariate Correlation ${ }^{\mathrm{a}}$ Matrix of Key Variables

\begin{tabular}{|c|c|c|c|c|c|c|c|c|c|c|c|c|c|c|c|}
\hline & $\mathbf{N}$ & 2 & 3 & 4 & 5 & 6 & 7 & 8 & 9 & 10 & 11 & 12 & 13 & 14 & 15 \\
\hline 1. Southeast university & 540 & .067 & .027 & .001 & .020 & -.016 & .002 & $.108^{* *}$ & $-.116^{* *}$ & .079 & -.004 & .028 & $.165^{* *}$ & $.134^{* *}$ & $.141^{* *}$ \\
\hline 2. Female & 540 & 1 & -.072 & $-.109^{* *}$ & -.065 & $-.161^{* * *}$ & $-.127^{* *}$ & -.037 & $.124^{* *}$ & -.068 & -.062 & $.129^{* *}$ & .084 & $-.126^{* *}$ & $.118^{* *}$ \\
\hline 3. SI & 540 & & 1 & $.494^{* *}$ & $.433^{* *}$ & $.508^{* *}$ & $.476^{* *}$ & $.572^{* *}$ & .043 & $.429^{* *}$ & $.353^{* *}$ & .000 & .079 & $.351^{* *}$ & $.259^{* *}$ \\
\hline 4. SP & 539 & & & 1 & $.602^{* *}$ & $.249^{* *}$ & $.343^{* *}$ & $.405^{* *}$ & $.099^{*}$ & $.202^{* *}$ & $.199^{* *}$ & .038 & $.102^{*}$ & $.202^{* *}$ & $.184^{* *}$ \\
\hline 5. SA & 531 & & & & 1 & $.278^{* *}$ & $.333^{* *}$ & $.317^{* *}$ & .086 & $.166^{* *}$ & .083 & .049 & $.189^{* *}$ & $.174^{* *}$ & $.224^{* *}$ \\
\hline 6. NSSI thoughts & 510 & & & & & 1 & $.740^{* *}$ & $.338^{* *}$ & .005 & $.285^{* *}$ & $.196^{* *}$ & -.024 & .082 & $.293^{* *}$ & $.225^{* *}$ \\
\hline 7. NSSI & 508 & & & & & & 1 & $.259^{* *}$ & .031 & $.203^{* *}$ & $.146^{* *}$ & .046 & .063 & $.238^{* *}$ & $.188^{* *}$ \\
\hline 8. SI/impulse severity & 499 & & & & & & & 1 & .078 & $.48^{* * *}$ & $.430^{* *}$ & .074 & $.171^{* *}$ & $.321^{* *}$ & $.238^{* *}$ \\
\hline 9. Fearlessness of death & 420 & & & & & & & & 1 & .055 & .088 & -.054 & -.025 & -.080 & -.002 \\
\hline 10. Depression/hopelessness & 410 & & & & & & & & & 1 & $.540^{* *}$ & $.133^{* *}$ & $.139^{* *}$ & $.417^{* *}$ & $.347^{* *}$ \\
\hline 11. Thwarted Belongingness & 406 & & & & & & & & & & 1 & .040 & $.096^{*}$ & $.343^{* *}$ & $.274^{* *}$ \\
\hline 12. Problems with alcohol & 406 & & & & & & & & & & & 1 & $.292^{* *}$ & .078 & $.212^{* *}$ \\
\hline 13. Problems with drugs & 407 & & & & & & & & & & & & 1 & $.155^{* *}$ & $.280^{* *}$ \\
\hline 14. Behavioral inhibition & 538 & & & & & & & & & & & & & 1 & $.529^{* *}$ \\
\hline 15. Emotional control & 538 & & & & & & & & & & & & & & 1 \\
\hline
\end{tabular}

Notes: NSSI = nonsuicidal self-injury, lifetime history; SA = suicide attempt, aborted attempt, or interrupted attempt, lifetime history; SD = standard deviation; $\mathrm{SI}=$ suicidal ideation, lifetime history; $\mathrm{SP}=$ suicide plan, lifetime history. ${ }^{\text {aPoint }}$ biserial correlations used for binary items.

$* p<.05 ; * * p<.01$ 
LATENT CLASS ANALYSIS OF COLLEGE STUDENT SITB

Table 4. Model fit statistics for 2-5 class solutions of self-injurious thoughts and behaviors among students $(\mathrm{N}=626)$

\begin{tabular}{cccccccc}
\hline Class Solution & LL & AIC & BIC & SSA-BIC & Entropy & $\begin{array}{c}\text { LMR-LRT } \\
\text { p-value }\end{array}$ & $\begin{array}{c}\text { BLRT } \\
\text { p-value }\end{array}$ \\
\hline 2 & -904.70 & 1831.40 & 1880.24 & 1845.31 & 0.89 & $<.001$ & $<.001$ \\
3 & -864.02 & 1762.04 & 1837.51 & 1783.54 & 0.93 & $<.001$ & $<.001$ \\
4 & -841.39 & 1728.78 & 1830.88 & 1757.86 & 0.92 & $<.001$ & $<.001$ \\
5 & -840.04 & 1738.07 & 1866.81 & 1774.74 & 0.94 & $\mathbf{0 . 0 1}$ & $\mathbf{0 . 6 0}$ \\
\hline
\end{tabular}

Note: $\mathrm{LL}=$ Log likelihood; AIC = Akaike's Information Criteria; BIC = Bayesian Information Criteria; SSA-BIC $=$ Sample size adjusted BIC; LMR-LRT = Lo-Mendell-Rubin Adjusted likelihood ratio test; BLRT = Bootstrapped likelihood ratio test. 
LATENT CLASS ANALYSIS OF COLLEGE STUDENT SITB

Table 5. Model classification diagnostics of the 3-class solution

\begin{tabular}{lrrrr}
\hline & $\boldsymbol{\pi}_{\mathbf{k}}$ & $\mathbf{m c a P}_{\mathbf{k}}$ & AvePP $_{\mathbf{k}}$ & OCC $_{\mathbf{k}}$ \\
\hline High SITB & 0.09 & 0.09 & 0.98 & 373.09 \\
High SI/NSSI-Low SA & 0.13 & 0.13 & 0.87 & 43.83 \\
Low SITB & 0.78 & 0.78 & 0.98 & 14.83 \\
\hline
\end{tabular}

Note: $\pi_{\mathrm{k}}=$ model estimated proportion for class $\mathrm{k} ; \mathrm{AvePP}_{\mathrm{k}}=$ average posterior probability for class $\mathrm{k} ; \mathrm{mcaP}_{\mathrm{k}}=$ modal class assignment proportion for class k; NSSI = nonsuicidal self-injury; $\mathrm{OCC}_{\mathrm{k}}=$ odds of correct classification; $\mathrm{SA}=$ suicide attempt; $\mathrm{SI}=$ suicidal ideation; $\mathrm{SITB}=$ selfinjurious thoughts and behaviors. 
LATENT CLASS ANALYSIS OF COLLEGE STUDENT SITB

Table 6. Associations between SITB classes and demographic variables (R3STEP), $\mathrm{n}=626$

\begin{tabular}{lccc}
\hline & High SITB v. Low SITB & High SI/NSSI-Low SA v. Low SITB & High SI/NSSI-Low SA v. High SITB \\
\hline University & $0.32(0.32)$ & $0.04(0.31)$ & $0.28(0.44)$ \\
Sex & $-0.95^{*}(0.42)$ & $-1.01^{*}(0.39)$ & $0.06(0.57)$ \\
Race & $0.21(0.40)$ & $0.48(0.37)$ & $-0.27(0.52)$ \\
Ethnicity & $0.77(0.48)$ & $0.58(0.48)$ & $0.18(0.64)$ \\
Age & $-0.12(0.11)$ & $0.18(0.10)$ & $-0.30 *(0.14)$ \\
\hline
\end{tabular}

Note: Values are estimates from the R3STEP logistic regression analyses with standard error in parentheses. ${ }^{*} \mathrm{p}<.05$; NSSI = nonsuicidal selfinjury; SA = suicide attempt; $\mathrm{SI}=$ suicidal ideation; SITB = self-injurious thoughts and behavior. 
Table 7. Means and Standard Error of Correlates Across SITB Classes

\begin{tabular}{|c|c|c|c|c|}
\hline Variable & High SITB & High SI/NSSI-Low SA & Low SITB & Overall Chi-square \\
\hline \multicolumn{5}{|l|}{ Suicide risk variables } \\
\hline SI/impulse severity & $0.63(0.11)$ & $0.36(0.07)$ & $0.04(0.01)$ & $74.36^{* * *}$ \\
\hline Fearlessness of death & $2.40(0.15)$ & $1.92(0.14)^{\mathrm{a}}$ & $2.05(0.04)^{\mathrm{a}}$ & 5.95 \\
\hline \multicolumn{5}{|l|}{ Psychological correlates } \\
\hline Depression/hopelessness & $1.71(0.17)^{\mathrm{a}}$ & $1.71(0.18)^{\mathrm{a}}$ & $0.76(0.05)$ & $53.59^{* * *}$ \\
\hline Thwarted belongingness & $1.52(0.25)^{\mathrm{a}}$ & $1.29(0.19)^{\mathrm{a}}$ & $0.61(0.05)$ & $25.98^{* * *}$ \\
\hline Problems with alcohol & $0.74(0.15)^{\mathrm{ab}}$ & $0.46(0.13)^{\mathrm{bc}}$ & $0.62(0.05)^{\mathrm{ac}}$ & 1.83 \\
\hline Problems with drugs & $0.71(0.22)^{\mathrm{a}}$ & $0.32(0.14)^{\mathrm{ab}}$ & $0.27(0.04)^{\mathrm{b}}$ & 4.43 \\
\hline \multicolumn{5}{|l|}{ Cognitive Correlates } \\
\hline Behavioral inhibition & $1.57(0.05)^{\mathrm{a}}$ & $1.48(0.05)^{\mathrm{a}}$ & $1.29(0.01)$ & $45.07^{* * *}$ \\
\hline Emotional control & $1.58(0.06)^{\mathrm{a}}$ & $1.56(0.06)^{\mathrm{a}}$ & $1.23(0.02)$ & $55.23^{* * *}$ \\
\hline
\end{tabular}

Note: the difference between any two areas is not statistically significant $(\mathrm{p}<0.05)$ if they have any letter in common. $* * * p<.001 ;$ NSSI $=$ nonsuicidal self-injury; SA = suicide attempt; SI = suicidal ideation; SITB = self-injurious thoughts and behaviors. 